\title{
User-Review Oriented Social Recommender System for Event Planning
}

\author{
Rahul Kumar Singh, Pardeep Singh, Gourav Bathla* \\ School of Computer Science, University of Petroleum and Energy Studies, Dehradun, Uttarakhand 248007, India
}

Corresponding Author Email: gbathla@ddn.upes.ac.in

\section{https://doi.org/10.18280/isi.250514}

Received: 14 June 2020

Accepted: 25 September 2020

\section{Keywords:}

sentiment analysis, recommender systems, social network, social contextual information, wedding planner

\begin{abstract}
Recommender system is used to suggest product or topic based on user's interest. Existing recommender system have focused on books, product, music etc. The problem in existing recommender system is that wedding/event based suggestions are not available. In the modern information era; storage, communication has been a challenge due to information veracity, volume, and velocity. Due to the constant and exponential growth of information, the utilization of information for context-oriented services is not productive. In this paper, a wedding planner recommender system framework has been proposed based on hybrid approach i.e., content based, collaborative filtering technique. The motive of proposed framework is to generate user-specific recommendations for different tasks related to the event specially wedding event, analyzed from the user comments on his social networking portal. Its main objective is to assist the user for organizing the events by suggesting specific vendors needed to arrange the event activities. Also, it would enhance the sales of location sensitive products in social commerce. The trial study conducted using a set of Facebook users is carried out to validate the proposed recommendation system framework. The success of the proposed framework is reported in terms of the level of user satisfaction achieved.
\end{abstract}

\section{INTRODUCTION}

The process of making recommendations has been widely used since many years in every aspect of life. Before the dawn of Internet, recommender systems were still there but in personalized form. For example, if a person wanted his daughter to get married, he took recommendations from match makers and family members according to suitability of his daughter. If anyone wanted to visit tourist places, he normally took recommendations from his friends who were known to that place or who have visited there earlier. Even while selecting the clothes, people ask opinion of their friends and family members [1]. As internet is becoming popular, users started to take recommendations from the web [2]. Recommender systems give both personalized and nonpersonalized suggestions to users. Personalized means give recommendations according to user's preferences such as: if a user wants to go to restaurant, he has to give his preferences about location, food and many more things beforehand for getting good recommendations of restaurants according to his taste [3]. In non-personalized recommendations, recommender systems provide recommendations according to the content: For example, news recommender system such as Google news, recommends users news similar to the news that user has been watching [4]. Recommendations are not something which user type in search engine and get the results; it is the search result which comes after matching the user's query. According to the user's interest, recommendations are provided. Recommender systems are the way to deal with the overload of information reflected in the increasing volume of information artifacts in the web [5]. Recommender systems analyze existing information on the user activities in order to predict future preferences. Every recommender system implements a different paradigm for generating recommendations in heterogeneous domain [6]. Recommender systems have been recognized as an important tool on web science and e-commerce applications. Many famous websites have been using recommender systems for improving their sales such as Netflix [7], a web-based movie rental service and Amazon, an e-commerce company. Netflix Prize contest was a well-known contest in the history of recommender systems, it offered 10 million US dollar for improving the collaborative filtering algorithm by $10.6 \%$ accuracy. Many approaches are used in recommender systems and each approach has its advantages and limitations. Currently, the following types of approaches are found in practice:

Collaborative filtering [8-10]: In the mid 1990's collaborative filtering algorithms were introduced. This approach is very successful approach among all. Recommender systems based on it recommend items according to the rating behavior of users. Ratings of the recommended items are given by the users similar to active user to whom recommendations are offered or similar to the items previously rated by the active user [11]. But there is a contradiction that two users may have similar preferences in one category but different preferences in another. This approach does not work efficiently in sparse datasets which denotes less number of ratings on the items. It suffers from Cold start problem that means there is no rating for newly added items and newly added users have not rated any item so far then how recommendations are possible for these kinds of users and items [12].

Content based filtering $[13,14]$ : recommends items to 
active user according to the content information of items.

Demographic filtering $[8,14]$ : considers the ratings of users who have same age, sex and location as active user has for the recommendations.

Knowledge based filtering [8]: Recommendations are based on the matching of user's need and set of options available. These recommender systems have knowledge about how a particular item meets the need of a particular user. For example, knowledge mined from user's profile will be helpful in recommendations.

Hybrid filtering methods [8, 15]: Hybrid denotes combination of two or more than two approaches. Hybrid approach is used to mitigate the limitations of individual approaches. Collaborative filtering approach can be combined with content based, knowledge based and demographic based approaches. Of these approaches collaborative filtering, due to its predictive nature by understanding the user needs, provides recommendation based on user preference. This approach has been the most successful and useful method from commercial usage. Amazon, Epinion adopted social recommender systems for improving the accuracy of their prediction

Social recommender systems: Since the introduction of the Tapestry systems by Goldberg, recommender systems have been in existence [10]. Social media has become famous now days. Eminent examples are social resource sharing sites: Delicious (Bookmarks), Flickr (Images), CiteULike (Bibliographic), Youtube (videos), Slashdot (information) and Social networking sites: MySpace (Music), Twitter (micro blogger), Epinion (Product Review), Flixter (movie review), Facebook, Linkedin. A social network models relationship between different users and information is exchanged between them according to their relationship. Data in Social network is usually shown with graphs and matrices. Social network is a set of nodes (actor or user) and edges (relationship between actors). As shown in Figure 1, Relationships between users are of two types: directional (marriage, cousins) and nondirectional (friendship, seller-buyer, and employer-employee) [5]. In the social recommendations: Tag recommendations, people recommendations, and content recommendations take place. In the social network, items can be social entities such as persons or group of persons.

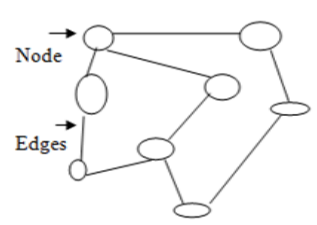

(a)

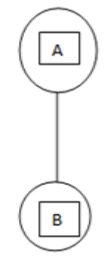

(b)

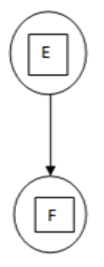

(c)
Figure 1. a) Social Network (Node-Actor, Edges-SocialRelationship), b) Non directional Relationship (marriage), c) Directional Relationship (Seller- Buyer)

Existing recommender system has not focused on wedding planning and social contextual information. Sparsity is issue faced by recommender system which is resolved by our proposed framework as social information is employed.

The significant contributions are specified below:

- As far as we know, this is the first recommender system framework for wedding planning.

- This framework utilizes knowledge based filtering; content based filtering, collaborative filtering, and demographic filtering for wedding planning tasks.

- A trial study has been performed on Facebook users to know their willingness for using this framework/application.

The rest of the paper organized as follows. Section 2 presents the literature survey of recommender systems and its relevance. In Section 3, the theoretical framework of wedding planner recommender system is proposed. Empirical study and results are presented in Section 4. Finally, Section 5 concludes the paper with future directions.

\section{LITERATURE SURVEY}

In a survey on recommender system presented by Adomavicius and Tuzhilin [8] various filtering approaches such as content based, collaborative and hybrid or detailed. The study indicates various generations in the recommender systems with merits and demerits that lead to possible extensions and upgrades. The research also details on modeling of users and activities for multiple criteria from a contextual prospective. These upgrades enhance the potential of recommender system and thereby make it more feasible for applications. Similar framework containing collaborative, content based along with demographic are used for an eatery based recommender system [14].

Similar research on recommender system evaluation conducted by Bobadilla et al. [9] and proposed three generation based evaluation based on pre-filtering approach. Initial method associated with optimization using vector distance $(\mathrm{KNN})$, Cumulative distance (aggregation) and diffusion specific to Single Value Decomposion (SVD) are collaborative filtering method that are memory dependent. Due to the challenges with storages and efficiency, hybrid approaches that used demographic filtering or content based filtering along with collaborative based filleting is recommended. There by the next generation of recommender system are developed based on algorithms that used hybrid approaches in social information analysis. The third generation of recommender system proposes to utilize personal information of any individual or entity along with hybrid algorithm supported for location and collaboration approaches. This generation of recommender system are heavily depended on internet as a primary means of communication for all types' information exchanges.

According to Schafer et al. [16], association of recommender system in retail industry through e-comers platform is increases at an exponential rate. Ben has also proposed different taxonomies based on various technological and platforms available for developers and customers. Similar work conducted by $\mathrm{Ma}$ et al. [17], that used social tags and blog post as primary source of contextual information is used for understanding the customer. Author propose to use probabilistic matrix factorization as a solution to improve recommendation accuracy by eliminating sparsity problem. Tan et al. [18] use a hyper graph model instead of graph model for representing objects and relationships. In Hyper graph, an edge can be connected with two or more than two nodes. Similarly, in social network, a group can have more than two or more than two users and a user can be a member of two or more than two groups. Chen et al. [19] proposed an approach that fully utilizes the social trust relationship by using the ensemble of probabilistic matrix factorization and social trust. 
Carrer-Neto et al. [20], presented second-generation recommender systems that use semantic web and social network information. They apply hybrid recommender systems on cinematographic domain and proves that hybrid approach provides accurate result.

Li et al. [21] proposed social commerce recommender that is based on similarity metrics, trust, reputation and social relationship. This recommender system improves the recommendations accuracy of traditional recommender system.

Kardan et al. [22] proposed a framework which allows targeted advertisements in online social network. Arazy et al. [23] introduced a framework that improves the accuracy of recommender systems and they explain four constructs such as homophily, trust, tie strength and social capital. This construct will impact the advice taking capability of recipients. Nilashi et al. [24], proposed a recommender system for tourism industry based on prediction methods, dimensionality reduction and clustering technique and achieve the significant accuracy. Bathla et al. [25], introduced a new technique based on transitive closure and hyperedge to improve social recommendations. Further, in order to personalize the agenda of users attending to a congress, Horowitz et al. [26] introduced EventAware recommender system. The author utilized the context-aware filtering and tag-based method to construct the context-aware mobile recommender system. Recently, Valcarce et al. [27] proposed a new embedding representation named prefs $2 \mathrm{vec}$ for memory-based recommender systems. This embedding model is inspired from word2vec embedding and utilized in collaborative filtering. Further, to recommend the suitable movie, Walek and Fojtik [28] proposed a new hybrid method named Predictory based on fuzzy expert system, content-based system and collaborative filtering system. Proposed system perform well as compare with traditional approach. Most recently, the performance of recommender system for largescale mixed complex data is improved by utilizing the deep neural network model [29].

\section{PROPOSED APPROACH}

Social recommender systems alleviate the sparsity problem that occurs in traditional recommender systems by incorporating trust, reputation, and credibility in social recommender systems. Evolution of social network is due to Self-interest, Homophiles, Trust/reputation, and sharing of resources. In this paper, a Wedding planner recommender system is proposed. Social information is used in our proposed framework to overcome sparsity and cold start issue. The reason for using social information is to understand the content to be suggested to users and wedding based information is used to understand the content of products to be recommended. Furthermore, factors such as time, location etc. are used to improve recommendation accuracy. This recommender system assists users with the recommendations of various assets that are the foundation stone of Wedding ceremony. Wedding planning is a very challenging task. In this recommender, Users (couple) will get recommendations of best invitation card shops, banquets, renowned jewelers, famous sweet shops, boutiques, photographers, saloons and many more things according to their location and their likings as shown in Figure 2. Other items like Honeymoon suites can also be recommended to the couples according to their interests and places they liked before. Contextual information such as location of a user is also incorporated into the system.

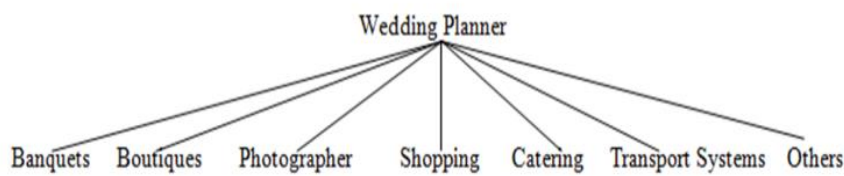

Figure 2. Taxonomy of Wedding Planner

In this paper, we propose a recommendations framework that gives recommendations to couple for their Wedding planning. A Social Recommender system based on this approach has certain mechanism for capturing the information of relationship between source of recommendations and recommendations seeker. Figure 3 represents an architecture of the proposed framework.

If a user named A post his status or life event Engaged with user named B in social network then Wedding planner recommender recommends various things that are of their interests. With this recommender, both party such as social commerce source and users (Couple) will take benefits. There are certain factors that can impact the performance of recommender system and these factors are listed as below:

\subsection{Factors}

When a company wants to improve sale, its first target is audience or we can say right audience who are really interested in its product. Posting an advertisement on social network is not enough, it is necessary to consider some factors that can have positive impact on sale.

Specific Group: Every recommendation is meant for some specific group means group who have an interest in the recommendations provided by recommender, so recommender system should consider that group. In Wedding planner system, recommendations that are shown to the couple should be according to them such as different banquets, boutiques, jeweler shop, Sweet shop, Card shop, Caterers, Transport systems, saloon and honeymoon suites. If these advertisements will be shown to a teenager or student, it will be of no use for them.

Location: If you have local business, you should target the local audience. For example, in Wedding planning, only local business comes into consideration because Banquet, sweet shop, and photographer recommendations will consider location of a couple. In Wedding planner, recommendations should be offered by considering home town location of both boy and girl because most of the time, Wedding is done either at boy's home town or girl's home town. So, recommender system by getting information regarding the location of boy or girl both from their profile will provide recommendations to them. This kind of recommendations will appeal to both the party.

Time: Recommendations will be given according to time. After posting the status of engaged by the users, recommender system will be started to show recommendations because in the mid time of engagement and Wedding, users started to do planning for their Wedding and at that time if they find recommendations what they actually looking for, they will be more satisfied. 


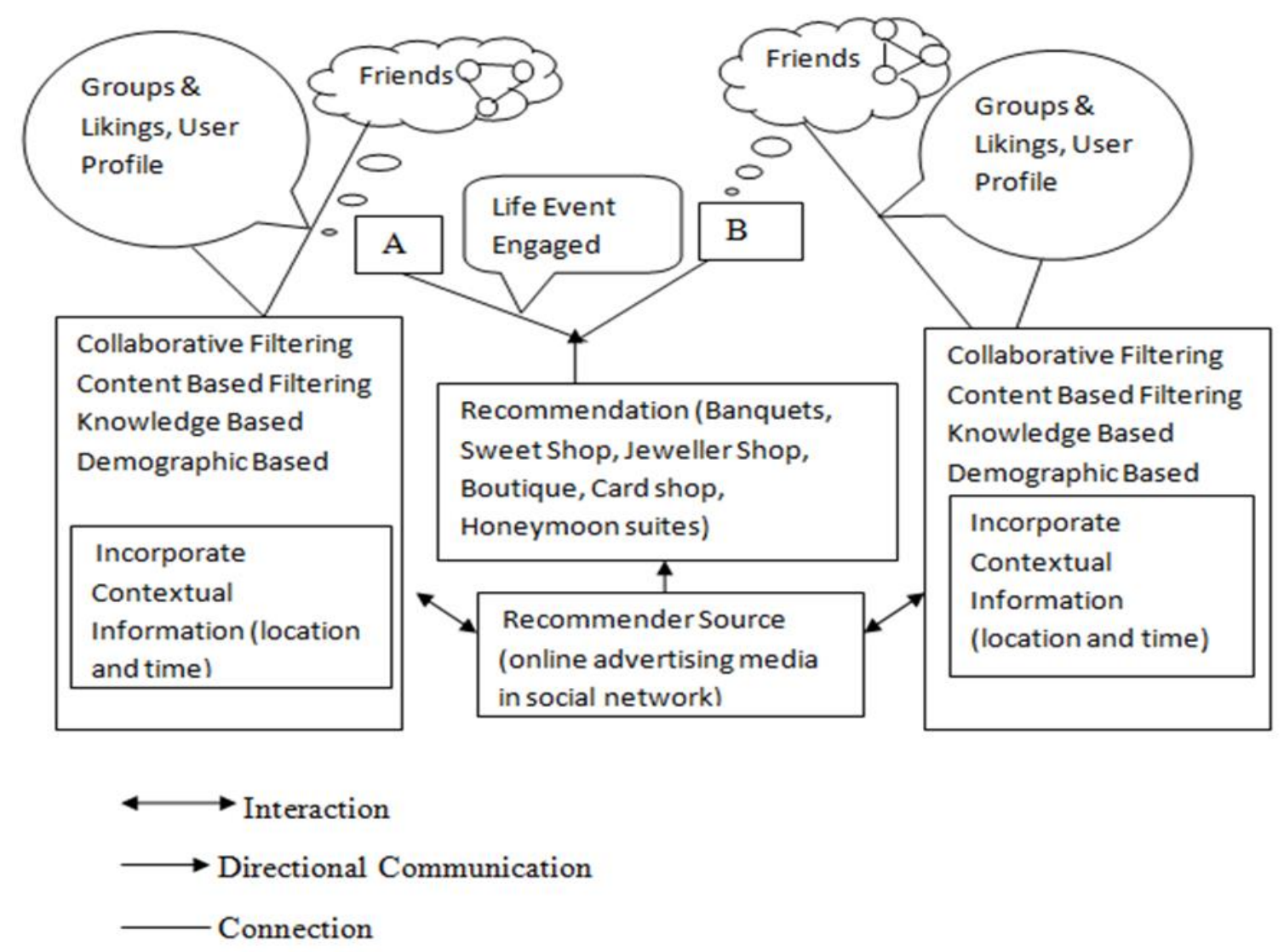

Figure 3. Proposed framework of wedding planning

Gender: Wedding planner system will take care of gender of users because recommendations vary gender to gender in some aspect. Wedding is the bond between the boy and girl. Some recommendations are gender oriented like a boutique recommendation for girl will be different than boy and another example is saloon.

Discount: The proposed Recommender system will show discount and special offers on the items that are recommended. Discount will attract the users.

\subsection{Wedding planner framework}

Wedding planner recommender system will follow a method that specifies how it will recommend right things to the right people in the social network. Wedding planner is a hybrid recommender system because it uses the knowledge based filtering; content based filtering, collaborative filtering, and demographic filtering. All these approaches will be used in it. These five steps are discussed below.

\footnotetext{
i. Check user's status (knowledge based)

ii. Check user preferences.

iii. Employ Hybrid (content based, collaborative filtering, and demographic) approach.

iv. Use social network (FOAF).

v. Employ reputation based on the ratings and social network analysis.

vi. Employ Social contextual information analysis.
}

Check User's Status (Knowledge Based): As shown in Figure 3, Two users have updated its profile by posting the status Engaged and knowledge based recommender will collect this information. Then this information is matched with the products that can be recommended to users. By mining the knowledge of engagement, recommender system will recommend different assets such as banquet, saloons, designers, photographers, honeymoon suites and many more items.

Check User Preferences: In proposed framework, we are taking two users who posted status engaged with each other in their social network and this is an initiating point for recommender system to turn on. Recommender system firstly checks user preferences then it will consider what the user's likings or interests and employ content based and collaborative filtering on it. In content based filtering, recommendations are according to user's profile and item description. Items are recommended to users according to their past preferences. Suppose if a user has liked one designer's costumes in past then content based recommender will show different costumes of the same designer. But user choices are not static, they are dynamic. One day they liked one designer's costumes another day they liked another's. This approach restricts the user to the same content and this problem is said overspecialization. To mitigate this problem collaborative filtering is also incorporated into system.

Hybrid Approach: We adopted hybrid approach in our framework. Collaborative filtering approach depends on the rating of users on items. Rating can be scalar on the scale of 1 to 5 and can be binary (like, dislike). Rating can be explicit and implicit. Explicit rating denotes rating given by user to an item and implicit denotes how many times a product is seen by user and for how much time, browsing history, click rate etc. In social network, binary rating is considered. This approach recommends an item that is preferred by users who are similar to the active user. Two type of similarities are considered first is user-user similarity and second is item-item similarity. In user-user similarity, if an active user has liked one place of visiting which are not yet seen by active user then these places 
will be recommended to the active user. In item-item similarity, if one item that is similar to another item that is liked by active user but not yet seen, will be recommended to him. For example: If an active user has liked one brand before, then similar brands (not the same brand) will be offered to him. But this approach is suffered from Cold start problem. If a user is new to the network and nothing is liked by him or item is newly added to the network and no one has liked it then how recommender will recommend items to users. To overcome the problems faced by individual approaches, content based and collaborative filtering is considered in combination. But sometimes this is not sufficient and other approaches are also combined. In demographic filtering, rating of those users is considered who share the same age, sex, and location with active user. If a user, who shared the same location with active user' has liked a banquet in past and active user is not aware of it then that banquet will be recommended to the active user. After employing these approaches, power of social network is utilized.

Employ Social Network Analysis: A user is connected with his friend, colleagues, family members via a network and that network is called social network where he can exchange messages, share data, post comments and can be member of any social group. User's information can be retrieved from user's profiles and user's behavior patterns. There are two types of relation in social network: one is direct relationship and other is indirect relationship. In the social network, there is a direct relationship between user and his friends and indirect relationship between user and his friend of friend by transitive property. If user $\mathrm{A}$ is the friend of user $\mathrm{B}$ and user $\mathrm{B}$ is the friend of user $C$ then user $A$ is indirectly friend of user $\mathrm{C}(\mathrm{A}->\mathrm{B}, \mathrm{B}->\mathrm{C}=>\mathrm{A}->\mathrm{C})$. Social network uses these kinds of relationships in recommendations. Social network become the part of daily life and a user wants to get recommendations on any product from his friend or family members to whom he trust the most. User depends on different users for different category of products. Trust plays a major role in social network. If a user gets recommendations from the source that satisfied him, he will trust that recommender source more than others. Trust is depending on whether user is taking recommendations or not. It tells the user likelihood of accepting the recommendations. Reputation is the measure of collective trustworthiness. Homophily specifies the similarity between users. If two users are similar then their interests will also be similar. Close Friends who share the strong tie means who interact more, are similar to each other. In proposed framework, Items liked by friends and trustworthy sources will be recommended to the users.

Employ Social Contextual Analysis: In Wedding planner system, Contextual Information such as location, season will also be employed. Suppose users (A, B) who are going to be married belong to Delhi and Mumbai. Then the most probably chance of their marriage happened in these two cities only. So, online advertisement business will recommend advertisements according to their cities. There can be possibility of not having online business in small cities. There are 29 states in India and in every state there are so many districts and in districts there are so many towns. If a user belongs to such place that does not have online business facilities then nearby place or city is recommended to him by using Google maps API. For example, if a user belongs to Rohtak where no online business facilities available then Delhi's online business companies will recommend their product.

\section{EMPIRICAL STUDY AND RESULTS}

Facebook is a large social networking site that is used by more than 800 million users per day. Representational State Transfer (REST) based communication is used by most of the web 2.0 applications. Facebook make new changes in its open API named Graph API which implement the open graph protocol and programming interface. Graph API gives number of objects to developers and with the help of these objects; they can search data of their interest. These objects contain public post, pages, groups, and events. As API is RESTful, these objects can be accessed through HTTP request, if developer has sufficient permission. Data is collected from these objects and these objects are parsed into JSON objects. Before collecting data, a pretest is performed on Facebook users. A questionnaire is prepared and asked Facebook users to fill in. An invitation link is sent through mail to 935 Facebook users who have posted their status "Engaged". Questionnaire contains questions regarding whether they will take recommendations from Facebook or not as shown in Table 1.

Table 1. Questions used in questionnaire

\begin{tabular}{|c|}
\hline Questions \\
\hline $\begin{array}{l}\text { Do you like to take item recommendation for your wedding so that } \\
\text { your Wedding preparation would be easy? }\end{array}$ \\
\hline $\begin{array}{l}\text { Do you like to take seasonal recommendation so that you can } \\
\text { enjoy your Wedding? }\end{array}$ \\
\hline $\begin{array}{l}\text { Do you like to take location based recommendation so that your } \\
\text { time will be saved and you can utilize your time in other important } \\
\text { work? }\end{array}$ \\
\hline Do you want to take item recommendations of your likings? \\
\hline $\begin{array}{l}\text { Do you like to take recommendations of those items that are liked } \\
\text { by your friends? }\end{array}$ \\
\hline $\begin{array}{l}\text { Do you want to save your money by getting items in cheaper rate } \\
\text { or in discounted price? }\end{array}$ \\
\hline $\begin{array}{l}\text { Do you want to get recommendations of new items (new in } \\
\text { market) for which you are not aware before? }\end{array}$ \\
\hline
\end{tabular}

Out of 935 Facebook users, 630 users answer the questions and 305 users did not give any response to questionnaire. Out of 630 users, 532 users answer positively and 98 users answer negatively as shown in Figure 4. A Facebook application named event planner is made and analyzes how many users like this application or visit the page. This application uses Google map API to track the location of users and location of vendors who will offer recommendations to users.

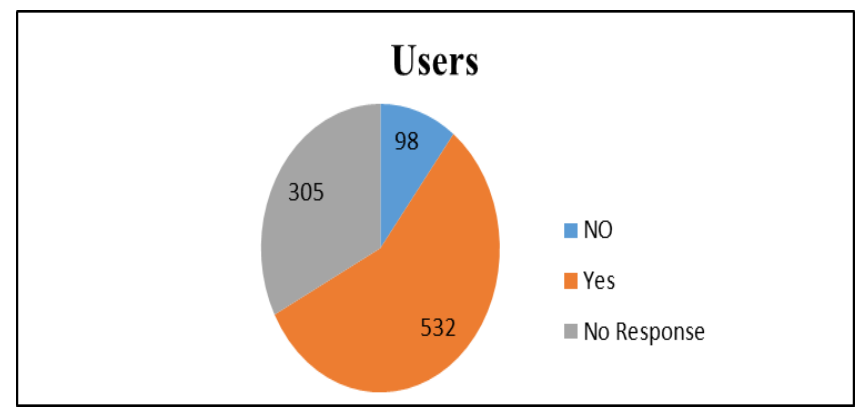

Figure 4. User distribution

Among 532 users, we pick 20 users and these users take recommendations according to factors. Out of 20 users, users take recommendations due to their likings or liked product, location based products and discounted items but ratio of these 
factors in recommendation is varied according to user choice. Users take recommendations with the combination of above stated factors such as 2 users take recommendations with the combination of likings, location, discount and seasonal factors in the ratio of 4.3, 2.4, 6 and 2 respectively as shown in Figure 5 .

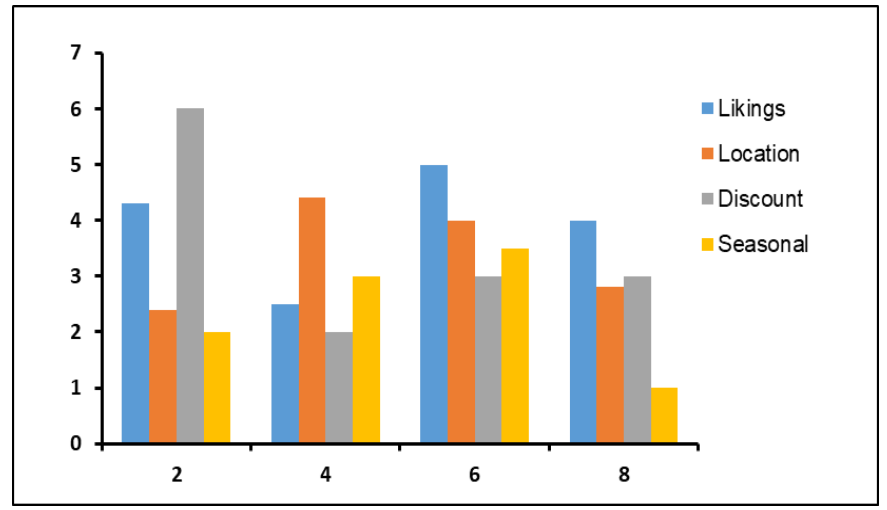

Figure 5. Recommendation taken by users according to factors

After giving recommendation to users, satisfaction of users is also considered. As satisfaction of user is subjected, we need to give focus on feedback given by user.

A Questionnaire has prepared for 20 users that measure satisfaction level of them. 16 questions are present in questionnaire as shown in Table 2 and each question has 6 options:
a) Strongly Agree
b) Agree
c) Neutral
d) Disagree
e) Strongly Disagree

Table 2. Questions used for measuring satisfaction of users

\begin{tabular}{|c|}
\hline Questions \\
\hline The products in the recommended list attract my attention. \\
\hline $\begin{array}{l}\text { The products in the recommended list also remind me others } \\
\text { product which slipped my memory. }\end{array}$ \\
\hline The products recommended are relevant to me. \\
\hline $\begin{array}{l}\text { The recommendations make me aware about usage of } \\
\text { recommended products that } \mathrm{i} \text { was not aware before. }\end{array}$ \\
\hline $\begin{array}{l}\text { Recommendations help me getting information related to } \\
\text { discounts and schemes. }\end{array}$ \\
\hline $\begin{array}{l}\text { If } \mathrm{i} \text { watch my favorite product in the recommendation list, there } \\
\text { are more chances of taking that recommendation. }\end{array}$ \\
\hline $\begin{array}{l}\text { mmendation because products in recommendation } \\
\text { g to my location. }\end{array}$ \\
\hline $\mathrm{n}$ list has seasonal products. \\
\hline $\begin{array}{l}\text { Recommendation helps me in saving time to search for the } \\
\text { products. }\end{array}$ \\
\hline Recommendation helps me comparing the various brands. \\
\hline Recommendation plays a role in switching the brands. \\
\hline Recommendation excited me to purchase unnecessary products. \\
\hline Recommendation is a good source of information. \\
\hline $\begin{array}{l}\text { The quality of most of the products in recommendation list met } \\
\text { my expectations. }\end{array}$ \\
\hline Recommendation helps me judging the current trends or fashions. \\
\hline made only for branded products. \\
\hline
\end{tabular}

Figure 6 depicts the user satisfaction level that is measured by taking the feedback from the users. $70 \%$ users are satisfied with the recommendation, $20 \%$ users are unsatisfied and $10 \%$ in dilemma. At Last, by measuring satisfaction level, it can be said that the proposed approach provides good quality recommendations.

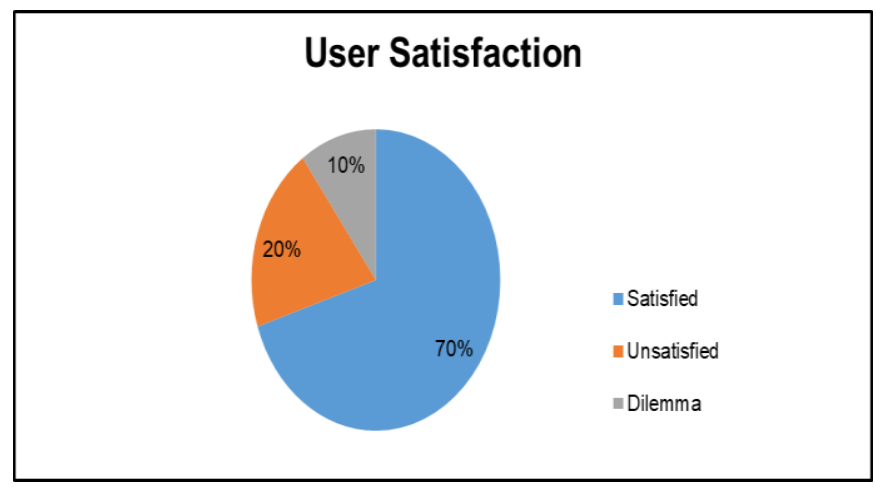

Figure 6. User satisfaction

\section{CONCLUSION AND FUTURE WORK}

In this paper, wedding planner recommender system has been proposed based hybrid approach i.e., content-based and collaborative filtering approach. An empirical study has been performed on 932 Facebook users in that 630 users actively participate with positive or negative responses. Among 532 positively respond users, we randomly pick 20 users and these users take recommendations according to factors. Based on questionnaire, satisfaction level of the users is measured. With the help of proposed framework, users can arrange their marriage without any burden. Online advertisement business can also get benefit out of it. In future, deep learning techniques will be employed to further improve recommendation accuracy. Moreover, evaluation metrics such as MAE and RMSE will be used to validate better recommendations.

\section{REFERENCES}

[1] Sharma, R., Vinayak, S., Singh, R. (2016). Guide me: A research work area recommender system. International Journal of Intelligent Systems and Applications, 8(9): 3037. https://doi.org/10.5815/ijisa.2016.09.04

[2] Singh, R.K., Sachan, M.K., Patel, R.B. (2020). 360 degree view of cross-domain opinion classification: A survey. Artificial Intelligence Review. https://doi.org/10.1007/s10462-020-09884-9

[3] Sharma, R., Singh, R. (2016). Evolution of recommender systems from ancient times to modern era: A survey. Indian Journal of Science and Technology, 9(20): 1-12. https://doi.org/10.17485/ijst/2016/v9i20/88005

[4] Inan, E., Tekbacak, F., Ozturk, C. (2018). Moreopt: A goal programming based movie recommender system. Journal of Computational Science, 28: 43-50. https://doi.org/10.1016/j.jocs.2018.08.004

[5] Singh, R., Rani, A. (2017). A survey on the generation of recommender systems. International Journal of Information Engineering \& Electronic Business, 9(3). https://doi.org/10.5815/ijieeb.2017.03.04

[6] Bathla, G., Aggarwal, H., Rani, R. (2020). Scalable recommendation using large scale graph partitioning 
with Pregel and Giraph. International Journal of Cognitive Informatics and Natural Intelligence (IJCINI), 14(4):

$42-61$. https://doi.org/10.4018/IJCINI.2020100103

[7] Koren, Y., Bell, R., Volinsky, C. (2009). Matrix factorization techniques for recommender systems. Computer, 42(8): https://doi.org/10.1109/MC.2009.263

[8] Adomavicius, G., Tuzhilin, A. (2005). Toward the next generation of recommender systems: A survey of the state-of-the-art and possible extensions. IEEE Transactions on Knowledge and Data Engineering, 17(6): 734-749. https://doi.org/10.1109/TKDE.2005.99

[9] Bobadilla, J., Ortega, F., Hernando, A., Gutiérrez, A. (2013). Recommender systems survey. KnowledgeBased Systems, 46: 109-132. https://doi.org/10.1016/j.knosys.2013.03.012

[10] Goldberg, D., Nichols, D., Oki, B.M., Terry, D. (1992). Using collaborative filtering to weave an information tapestry. Communications of the ACM, 35(12): 61-70. http://dx.doi.org/10.1145/138859.138867

[11] van Capelleveen, G., Amrit, C., Yazan, D.M., Zijm, H. (2019). The recommender canvas: A model for developing and documenting recommender system design. Expert Systems with Applications, 129: 97-117. https://doi.org/10.1016/j.eswa.2019.04.001

[12] Singh, R.K., Rani, A., Sachan, M.K. (2017). Fuzzy automata: A quantitative review. https://www.researchgate.net/publication/322064617 F uzzy_Automata_A_Quantitative_Review.

[13] Salter, J., Antonopoulos, N. (2006). CinemaScreen recommender agent: combining collaborative and content-based filtering. IEEE Intelligent Systems, 21(1): 35-41. http://dx.doi.org/10.1109/MIS.2006.4

[14] Pazzani, M.J. (1999). A framework for collaborative, content-based and demographic filtering. Artificial Intelligence Review, 13(5-6): 393-408. https://doi.org/10.1023/A:1006544522159

[15] Burke, R. (2002). Hybrid recommender systems: Survey and experiments. User Modeling and User-Adapted Interaction, $\quad 12(4)$ : 331-370. https://doi.org/10.1023/A:1021240730564

[16] Schafer, J.B., Konstan, J., Riedl, J. (1999). Recommender systems in e-commerce. In Proceedings of the 1st ACM Conference on Electronic Commerce, pp. 158-166. https://doi.org/10.1145/336992.337035

[17] Ma, H., Zhou, T.C., Lyu, M.R., King, I. (2011). Improving recommender systems by incorporating social contextual information. ACM Transactions on Information Systems (TOIS), 29(2): 1-23. https://doi.org/10.1145/1961209.1961212

[18] Tan, S., Bu, J., Chen, C., Xu, B., Wang, C., He, X. (2011). Using rich social media information for music recommendation via hypergraph model. ACM
Transactions on Multimedia Computing, Communications, and Applications (TOMM), 7(1): 1-22 https://doi.org/10.1145/2037676.2037679

[19] Chen, C., Zeng, J., Zheng, X., Chen, D. (2013). Recommender system based on social trust relationships. In 2013 IEEE 10th International Conference on eBusiness Engineering, Coventry, UK, pp. 32-37. https://doi.org/10.1109/ICEBE.2013.5

[20] Carrer-Neto, W., Hernández-Alcaraz, M.L., ValenciaGarcía, R., García-Sánchez, F. (2012). Social knowledge-based recommender system. Application to the movies domain. Expert Systems with Applications, 39(12): https://doi.org/10.1016/j.eswa.2012.03.025

[21] Li, Y.M., Wu, C.T., Lai, C.Y. (2013). A social recommender mechanism for e-commerce: Combining similarity, trust, and relationship. Decision Support Systems, 55(3): 740-752. https://doi.org/10.1016/j.dss.2013.02.009

[22] Kardan, A.A., Hooman, M. (2013). Targeted advertisement in social networks using recommender systems. In 7th International Conference on e-Commerce in Developing Countries: With Focus on e-Security, pp. 1-13. https://doi.org/10.1109/ECDC.2013.6556728

[23] Arazy, O., Kumar, N., Shapira, B. (2009). Improving social recommender systems. IT Professional, 11(4): 3844. https://doi.org/10.1109/MITP.2009.76

[24] Nilashi, M., Bagherifard, K., Rahmani, M., Rafe, V. (2017). A recommender system for tourism industry using cluster ensemble and prediction machine learning techniques. Computers \& Industrial Engineering, 109: 357-368. https://doi.org/10.1016/j.cie.2017.05.016

[25] Bathla, G., Aggarwal, H., Rani, R. (2020). A graph-based model to improve social trust and influence for social recommendation. The Journal of Supercomputing, 76(6): 4057-4075. https://doi.org/10.1007/s1 1227-017-2196-2

[26] Horowitz, D., Contreras, D., Salamó, M. (2018). EventAware: A mobile recommender system for events. Pattern Recognition Letters, 105: 121-134. https://doi.org/10.1016/j.patrec.2017.07.003

[27] Valcarce, D., Landin, A., Parapar, J., Barreiro, Á. (2019). Collaborative filtering embeddings for memory-based recommender systems. Engineering Applications of Artificial Intelligence, 85: 347-356. https://doi.org/10.1016/j.engappai.2019.06.020

[28] Walek, B., Fojtik, V. (2020). A hybrid recommender system for recommending relevant movies using an expert system. Expert Systems with Applications, 158: 113452. https://doi.org/10.1016/j.eswa.2020.113452

[29] Bathla, G., Aggarwal, H., Rani, R. (2020). AutoTrustRec: Recommender system with social trust and deep learning using autoEncoder. Multimedia Tools and Applications, 79: 1-16. https://doi.org/10.1007/s1 1042-020-08932-4 